\title{
Endoprótesis intestinal recubierta para manejo de fístula enteroatmosférica, reporte de caso y revisión de la literatura
} Covered intestinal endoprosthesis for the management of enteroatmospheric fistula, case
report and review of the literature Ediel O. Dávila-Ruiz*, Roberto A. García-Manzano, Alan Barker-Antonio, Elías Hernández-Cruz y Emmanuel López-Martínez

Servicio de Cirugía General, Hospital Regional de Alta Especialidad de Oaxaca, Secretaría de Salud Oaxaca, San Bartolo Coyotepec, Oaxaca, México

\begin{abstract}
Resumen
El manejo de la fístula es un desafío para los cirujanos. Las fístulas enteroatmosféricas (EAF) se caracterizan por ser superficiales, de alto gasto y rodeadas de vísceras o tejido de granulación, con un control deficiente de su excreción debido a su anatomía. Describimos un enfoque alternativo para el manejo de la fístula enteroatmosférica, en un paciente con un abdomen hostil y resección intestinal masiva, seleccionándolo para la colocación de stent como medida de rescate, permitiendo el control de la fístula y el inicio de la alimentación entérica, lo que mejora sustancialmente la calidad de vida,
\end{abstract}

Palabras clave: Fístula enteroatmosférica. Stent intestinal. Fístulas enterocutáneas. Endoprótesis intestinal. Abdomen hostil.

\begin{abstract}
The management of fistulas is a challenge for surgeons, the enteroatmospheric fistulas are characterized by being superficial, high debit and surrounded by viscera or granulation tissue, with poor control of its excretion due to their anatomy. We describe an alternative approach to the management of enteroatmospheric fistulas, in a patient with a hostile abdomen and massive intestinal resection, selected for placement of a stent, as a rescue measure. Use of intestinal stent allowed fistula control and enteric feeding capacity, that substantially improves the quality of life,
\end{abstract}

Keywords: Enteroatmospheric fistula. Intestinal sten. Enterocutaneous fistulas. Intestinal endoprosthesis. Hostile abdomen.

\section{Introducción}

El manejo de las fístulas es un reto para los cirujanos desde que se iniciaron las intervenciones quirúrgicas en el tracto gastrointestinal. Existen múltiples variantes de fístulas y diversos tratamientos. En la actualidad, el manejo conservador es de elección en las fístulas de bajo gasto, lo cual permite el cierre espontaneo, evitando intervenciones quirúrgicas. Cuando el tratamiento conservador falla o no es posible, por fístulas de alto gasto como las enteroatmosféricas, se deben buscar nuevas técnicas que permitan el manejo y el cierre de la fístula.

\section{Correspondencia:}

*Ediel O. Dávila-Ruiz

Aldama, $\mathrm{s} / \mathrm{n}$ 
Las fístulas enteroatmosféricas son un subgrupo de las fístulas enterocutáneas que se caracterizan por ser superficiales, con alto gasto, rodeadas de vísceras o tejido de granulación, lo que causa mal control del gasto y lleva a malnutrición grave en periodos cortos debido a su alto gasto, alteraciones electrolíticas y sepsis ${ }^{1-3}$.

Si una fístula enteroatmosférica ocurre en el contexto de un abdomen abierto congelado se clasifica como Björck 4 y la mortalidad es del $42 \%$, la cual se ha reducido gracias a los avances en técnicas quirúrgicas y cuidados intensivos ${ }^{4}$.

Una fístula enteroatmosférica puede generarse en un contexto de síndrome de intestino corto, lo cual empeora el pronóstico del paciente. El síndrome de intestino corto se define como la condición resultante de la resección quirúrgica o de una enfermedad congénita, con incapacidad para mantener el balance energético, hidroelectrolítico y de micronutrientes con una dieta normal, necesitando suplementación intravenosa o parenteral. Esta complicación se puede presentar en un $15 \%$ de los pacientes sometidos a resección intestinal, un $75 \%$ de los pacientes sometidos a resecciones masivas y un $25 \%$ de los pacientes sometidos a múltiples resecciones. Los pacientes con menos de $180 \mathrm{~cm}$ de intestino están en riesgo de sufrirlo ${ }^{5,6}$.

En este caso se hace énfasis en la necesidad de restaurar el tránsito intestinal, con manejo del gasto de la fístula enteroatmosférica mediante el uso de una endoprótesis intestinal como medida de salvamento ${ }^{7,8}$. Esta medida es controversial, ya que existen distintos resultados, pero puede ser un tratamiento de rescate en pacientes protocolizados, en los cuales no existe posibilidad de más resecciones o cirugías intestinales, por abdomen hostil, enfermedad adherencial grave o intestino corto ${ }^{9,10}$.

Describimos un manejo alternativo de una fístula enteroatmosférica en un paciente con abdomen abierto Björck 4, en el contexto de abdomen congelado y resección intestinal masiva. Se llevó a cabo una búsqueda detallada del uso de endoprótesis como medida de rescate, para ofrecer un procedimiento que pudiera favorecer el control y cierre temprano de la fístula, mediante colocación de endoprótesis intestinal. Se utilizaron los términos MeSH: enteroatmospheric fistula, intestinal stent, enterocutaneous fistula, intestinal endoprosthesis y hostile abdomen.

\section{Caso clínico}

Varón de 47 años, agricultor, originario de Oaxaca, quien fue referido de un hospital de segundo nivel con

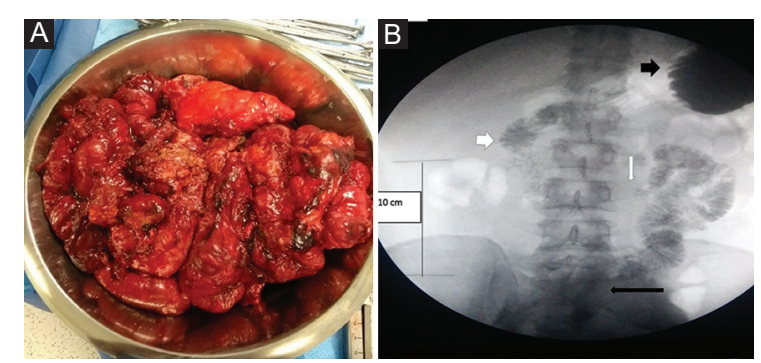

Figura 1. A: intestino resecado. B: medición del remanente de intestino, estómago (flecha negra corta), duodeno (flecha blanca corta), ángulo de Treitz (flecha blanca larga) y fuga de medio de contraste en el yeyuno (flecha negra larga).

diagnóstico de obstrucción intestinal. Antecedentes personales: hipertenso de 15 años de evolución en tratamiento con antagonista de los receptores de la angiotensina II. Antecedentes quirúrgicos: apendicetomía de urgencia hace 15 años con resolución favorable y laparotomía exploradora hace 5 años por apuñalamiento, sin complicaciones.

Inicia su padecimiento el 15 de febrero de 2018 con dolor abdominal, vómito, náusea e incapacidad para evacuar. Dos días después acude a un hospital de segundo nivel, se le diagnostica obstrucción intestinal y se le realiza laparotomía exploradora, con hallazgos de necrosis de $10 \mathrm{~cm}$ de yeyuno, por lo que se realizan resección y anastomosis término-terminal. Con mala evolución, se opera al sexto día por obstrucción, pero solo se realiza laparotomía con toma de biopsia y se le envía a un hospital de tercer nivel para protocolo diagnóstico, inicio de nutrición parenteral total y manejo de la obstrucción intestinal. Es sometido a una nueva intervención el día 7 de marzo por salida de material intestinal en el tercio inferior de la herida quirúrgica, y se evidencian múltiples laceraciones en cuerda de violín en la pared antimesentérica del intestino, secundarias a una lesión traumática por el material utilizado (polipropileno) para el cierre de la aponeurosis en la cirugía previa, además de adherencias Zulkhe II-III. Se decide la resección en bloque del intestino dañado (Fig. 1), dejando únicamente un remanente de yeyuno a $40 \mathrm{~cm}$ desde el ángulo de Treitz y la totalidad del colon. Se realizó una anastomosis yeyuno-colónica con engrapadora GIA $80 \mathrm{mmHg}$ y se cerró la cavidad abdominal. El manejo de la sepsis fue acorde a las guías actuales.

En el tercer día posquirúrgico el paciente presenta fuga de material intestinal y es necesario intervenir quirúrgicamente, con los siguientes hallazgos: enfermedad adherencial grave Zulkhe IV, imposibilidad de disecar el 


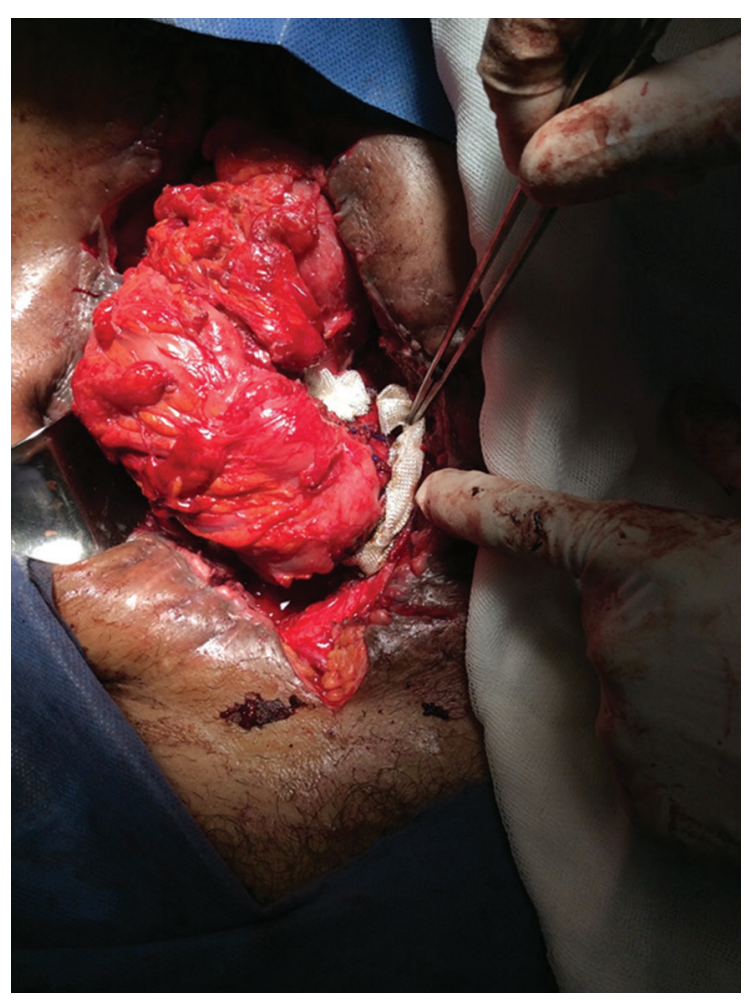

Figura 2. Se observa yeyuno-tranverso anastomosis.

yeyuno por adherencias firmes al sigmoides, y necrosis de la cara anterior del ciego. Se disecan los bordes necróticos, se remodela la anastomosis y se colocan drenajes en las caras anterior y posterior de la anastomosis. En el posoperatorio mediato se evidencia fuga, y debido a la imposibilidad de tratamiento quirúrgico se decide tratarla como fístula de alto gasto. Se inicia terapia antisecretora con omeprazol $80 \mathrm{mg}$ al día en infusión y loperamida $16 \mathrm{mg} / \mathrm{día}$, además de continuar con nutrición parenteral $2400 \mathrm{kcal} /$ día y suplementación multivitamínica. Se realiza tránsito intestinal con medio de contraste hidrosoluble para determinar la cantidad de intestino remanente, siendo aproximadamente de $40 \mathrm{~cm}$ desde el ángulo de Treitz (Fig. 1). Además, se realiza estudio en colon por enema con medio hidrosoluble, verificando la permeabilidad del tracto inferior.

Se decide una tercera intervención en el hospital de tercer nivel, el 9 de abril, desmantelando la anastomosis previa, la cual presentaba dehiscencia de más del $70 \%$. Se decide hemicolectomía derecha con la finalidad de prevenir fugas debido a la pobre irrigación del remanente de ciego y de colon ascendente. Se realiza anastomosis transverso-yeyuno. No es posible separar el yeyuno adherido al colon sigmoides (Fig. 2). Al cuarto día se produce fuga de la anastomosis por acodamiento en el colon sigmoides y aumento de la

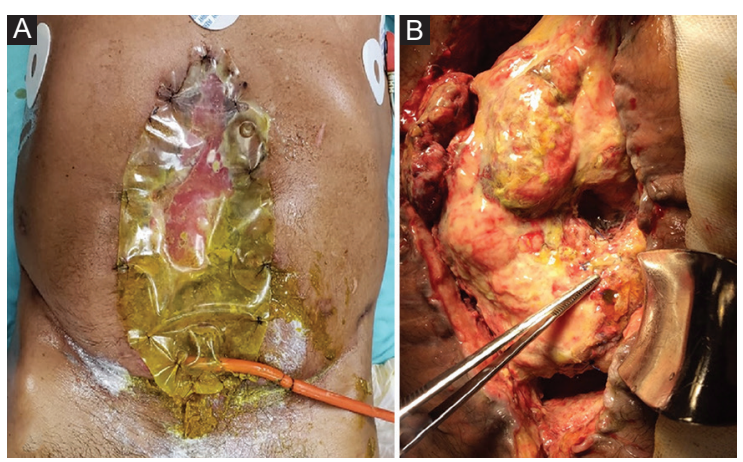

Figura 3. A: pobre control de la fuga. B: dehiscencia de la cara anterior de la anastomosis.

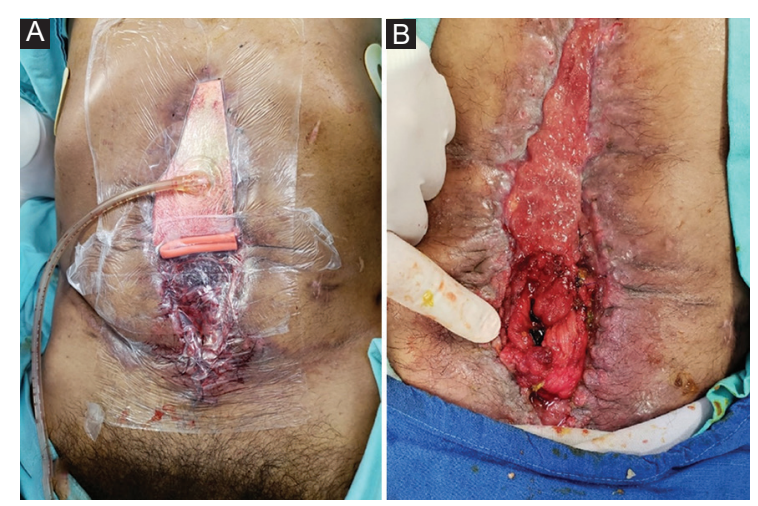

Figura 4. A: colocación de sistema VAC. B: fístula enteroatmosférica aislada.

presión (Fig. 3). Para limitar la contaminación de la cavidad, se coloca un sistema de cierre asistido por presión (VAC) (Fig. 4), lo cual permite el cierre de la línea media de la herida en cuatro sesiones de VAC, dejando únicamente la dehiscencia de anastomosis como una fístula enteroatmosférica aislada. Debido a la condición del paciente (desnutrición, sepsis, desequilibrio hidroelectrolítico, abdomen hostil) no son posibles más cirugías, por alto riesgo de perforación y desvascularización. El aislamiento de la fístula ayudó a mejorar el estado hemodinámico y el control de la sepsis; sin embargo, las pérdidas entéricas eran elevadas, lo cual perpetuaba el desequilibrio hidroelectrolítico, la desnutrición y la malabsorción ${ }^{11-14 .}$

\section{Técnica quirúrgica de colocación de endoprotesis intestinal e indicaciones}

En el contexto del paciente con fístula enterocutánea y síndrome de intestino corto se propone la colocación de una endoprótesis recubierta de $36 \times$ 


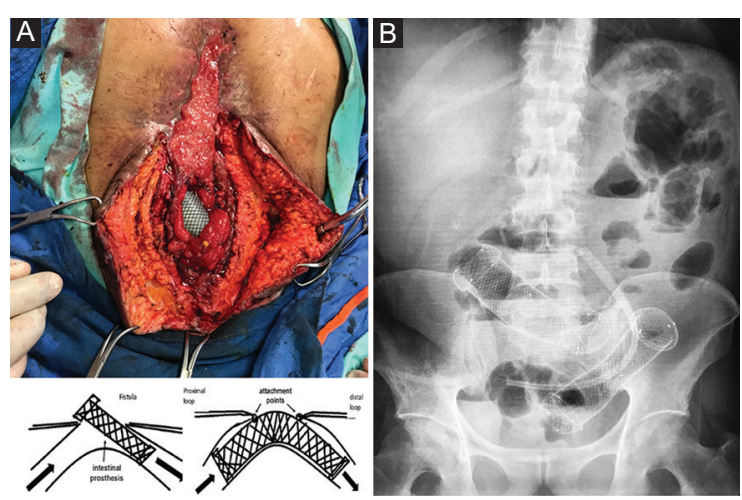

Figura 5. A: colocación y ajuste de la endoprótesis intestinal. B: radiografía de control tras la colocación del stent en el sitio de la fístula y sigmoides.

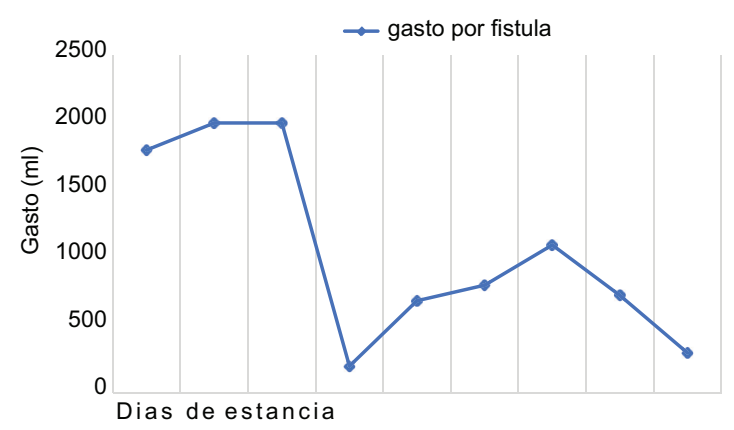

Figura 6. Gasto por la fístula. A: colocación de la endoprótesis; B: inicio de la dieta enteral; C: control de la fístula y disminución del gasto; D: alta hospitalaria.

$100 \mathrm{~mm}$ (nitinol) para restituir el tránsito intestinal desde el asa distal (yeyuno) a proximal (colon transverso), permitiendo el control de la fístula, la granulación desde los bordes intestinales y el cierre definitivo. La intervención se realizó el día 16 de mayo, colocando la endoprótesis a través de la línea media invaginando los dos segmentos del stent y fijándolo con puntos de sutura no absorbible. Mediante colonoscopia transoperatoria se colocó un segundo stent en el sigmoides para reducir la presión intraluminal y prevenir fugas ${ }^{9,10}$ (Fig. 5). Después de la colocación de los stents disminuyó el gasto de la fístula, de 1500 a $200 \mathrm{ml}$, en un periodo de 5 días. Se inició la alimentación enteral, coincidiendo con un incremento del gasto, el cual se estabilizó hasta $300 \mathrm{ml}$. El paciente continuó con nutrición parenteral total a razón de $30 \mathrm{kcal} / \mathrm{kg}$ al día (Fig. 6). Al noveno día posquirúrgico se decide el alta, sin evidencia de sepsis ni de desequilibrio hidroelectrolítico, y con control de la fístula.

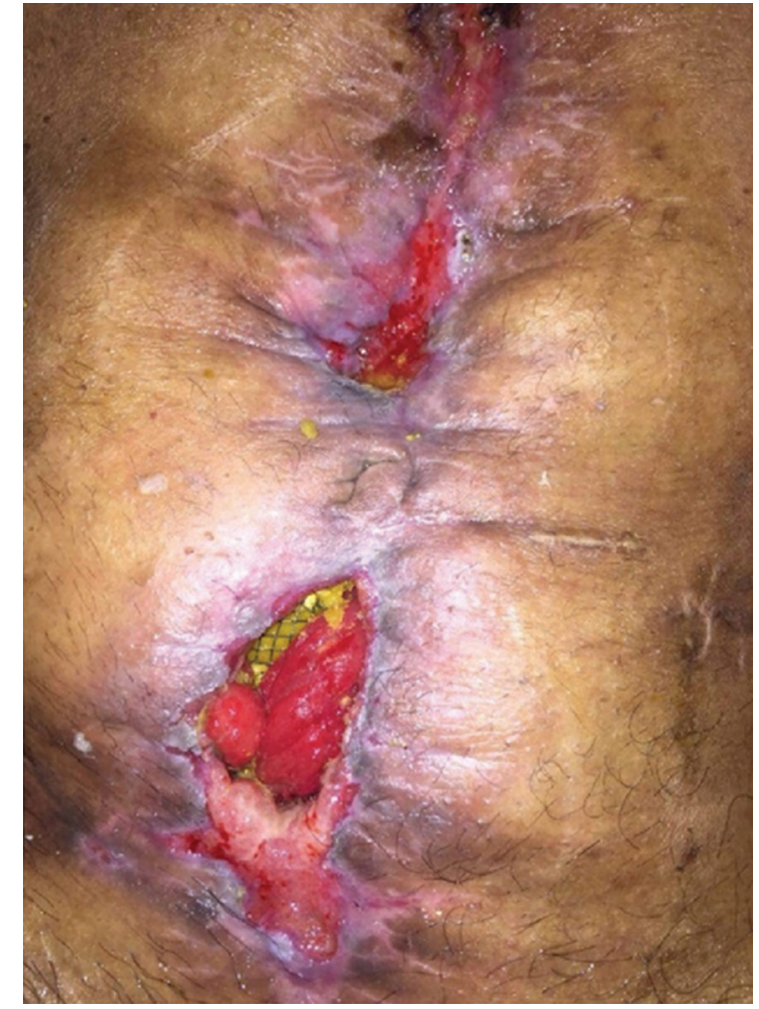

Figura 7. Fístula en proceso de cierre por segunda intención. Se observa el stent en el tercio medio.

\section{Resultados}

Se realizó seguimiento por consulta externa con el equipo multidisciplinario de manera mensual, evaluando el estado nutricional y la herida quirúrgica, que se encontraba cerrada en un $90 \%$ a los 6 meses de seguimiento, sin necesidad de nutrición parenteral total, solo suplementación multivitamínica y dieta sin oxalatos, y el gasto a través de la herida era de 50 $150 \mathrm{ml}$ (Fig. 7). El paciente se reincorporó de manera parcial a sus actividades diarias y se valía por sí mismo.

El uso de la endoprótesis permitió el inicio temprano de la alimentación enteral, mejorando la calidad de vida y disminuyendo las complicaciones relacionadas con la nutrición parenteral total (daño hepático, esteatosis, síndrome colestásico, infecciones). La adaptación final del colon se alcanza a los 2 años. El paciente no presentó molestias abdominales en el sitio del stent.

\section{Discusión}

El uso de endoprótesis intestinales fue probado en fístulas colocutáneas relacionadas con estomas por 
Tabla 1. Indicaciones y beneficios de las endoprótesis en las fístulas enterocutáneas

\begin{tabular}{ll}
\hline Indicaciones & Beneficios \\
\hline $\begin{array}{l}\text { Fistulas colocutáneas } \\
\text { relacionadas con estomas }\end{array}$ & $\begin{array}{l}\text { Aislamiento de la herida, favorece la } \\
\text { granulación y el cierre por segunda } \\
\text { intención }\end{array}$ \\
$\begin{array}{ll}\text { Control de la materia fecal } \\
\text { (disminución de la carga } \\
\text { bacteriana), disminución de } \\
\text { infecciones }\end{array}$ & $\begin{array}{l}\text { temprana, disminuye la } \\
\text { translocación bacteriana, mejora la } \\
\text { salud del enterocito }\end{array}$ \\
$\begin{array}{ll}\text { Abdomen hostil o congelado, } \\
\text { o incapacidad de movilizar los }\end{array}$ & $\begin{array}{l}\text { Tolerada simple, reproducible y bien } \\
\text { intestinos }\end{array}$ \\
$\begin{array}{l}\text { Necesidad de suipo multidisciplinario) } \\
\text { oral }\end{array}$ & $\begin{array}{l}\text { Capacidad de recolocación en caso } \\
\text { Restitución del tránsito }\end{array}$ \\
intestinal & \\
\hline
\end{tabular}

Nikfarjam et al. ${ }^{9}$ en dos pacientes, permitiendo limitar la contaminación fecal en la herida y el cierre de la fístula. Puig et al. ${ }^{15}$, de la Clínica Mayo, utilizaron endoprótesis para el manejo de fístulas y estenosis en anastomosis de cirugía bariatrica, lo cual permitió la remisión de la sepsis, mejorando el estado nutricional antes de la corrección quirúrgica definitiva.

En 2016, Melich et al..$^{10}$ reportaron el control endoscópico de fístulas enterocutáneas mediante la intususcepción de dos stents a través de la misma fístula, permitiendo su cierre con resultados aceptables. Xu et al. ${ }^{16}$ reportaron un caso de fístula enteroatmosférica manejado con un stent impreso en 3D, obteniendo una reducción drástica en el gasto de la fístula y una recuperación rápida.

El uso de prótesis intestinales para la restitución del tránsito intestinal en pacientes seleccionados con fístula enterocutánea es un procedimiento de salvamento que puede ofrecerse después de un estudio minucioso por un equipo multidisciplinario, y es efectivo para la restitución rápida del tránsito intestinal, en el contexto de abdomen hostil, sin posibilidad de movilización de segmentos intestinales, cirugías de elongación o resección intestinal, siendo necesario en pacientes en quienes debe maximizarse el remanente de intestino funcional.

La colocación de endoprótesis permite iniciar la vía enteral, mejora la salud general del paciente y disminuye los requerimientos de nutrición parenteral y las complicaciones de esta ${ }^{17}$.

Rebibo et al. ${ }^{18}$ explicaron el uso de endoprótesis cubierta como tratamiento de la fístula enteroatmosférica
Tabla 2. Riesgos, complicaciones y consideraciones del uso de endoprótesis enterocutáneas para el manejo de fístulas

\begin{tabular}{|c|c|}
\hline Riesgos y complicaciones & Consideraciones \\
\hline $\begin{array}{l}\text { Fibrosis del segmento } \\
\text { intestinal en contacto con la } \\
\text { endoprótesis }\end{array}$ & $\begin{array}{l}\text { Requiere fijación al tejido subcutáneo } \\
\text { y la piel }\end{array}$ \\
\hline $\begin{array}{l}\text { Necesidad de resección del } \\
\text { intestino fibrosado }\end{array}$ & $\begin{array}{l}\text { Necesidad de disminuir la } \\
\text { consistencia de las heces con } \\
\text { laxantes o fibra para reducir el riesgo } \\
\text { de migración y expulsión }\end{array}$ \\
\hline $\begin{array}{l}\text { Puede actuar como cuerpo } \\
\text { extraño y alterar la curación }\end{array}$ & $\begin{array}{l}\text { Necesidad de protección de la piel, } \\
\text { para promover la granulación y el } \\
\text { cierre de la fístula }\end{array}$ \\
\hline \multirow[t]{2}{*}{$\begin{array}{l}\text { Necesidad de } \\
\text { seguimiento mensual con } \\
\text { colonoscopia para detectar } \\
\text { complicaciones tempranas }\end{array}$} & $\begin{array}{l}\text { Una vez que el defecto se cierra, la } \\
\text { endoprótesis debe retirarse. Puede } \\
\text { resecarse el segmento intestinal o } \\
\text { quitar la fijación y observación hasta } \\
\text { su expulsión }\end{array}$ \\
\hline & $\begin{array}{l}\text { Se recomienda al menos } 2 \text { meses de } \\
\text { observación antes de tratar el retiro }\end{array}$ \\
\hline
\end{tabular}

asociada a ileostomía, mediante una técnica combinada endoscópica y de cirugía abierta, permitiendo el cierre de las fístula. Sin embargo, se necesitan más series de casos y un mayor número de pacientes para evaluar el riesgo-beneficio de este tratamiento.

Las indicaciones y los beneficios del uso de endoprótesis se resumen en la tabla 1. Los riesgos, las complicaciones y las consideraciones se resumen en la tabla $28,9,15,16$.

\section{Conclusiones}

El uso de endoprótesis para el control del gasto de fístulas enteroatmosféricas es controversial; sin embargo, se puede considerar como medida de salvamento en pacientes seleccionados, sobre todo en aquellos con altas posibilidades de muerte a causa de la fisiopatología de la fístula. Esto hace de las endoprótesis intestinales una medida aceptable para pacientes con esta patología. Se necesitan más series de casos tratados de esta manera y con un protocolo formal capaz de reproducir la técnica y evaluar el riesgo-beneficio.

\section{Agradecimientos}

Agradecemos a la institución HRAEO, a sus directivos y al personal que labora en ella, por su apoyo en esta publicación, y en especial a su equipo de cirugía. 


\section{Financiamiento}

Los autores declaran no recibir financiamiento para la realización de este artículo.

\section{Conflicto de intereses}

Para el presente artículo no existe conflicto de intereses.

\section{Responsabilidades éticas}

Protección de personas y animales. Los autores declaran que para esta investigación no se han realizado experimentos en seres humanos ni en animales.

Confidencialidad de los datos. Los autores declaran que han seguido los protocolos de su centro de trabajo sobre la publicación de datos de pacientes.

Derecho a la privacidad y consentimiento informado. Los autores han obtenido el consentimiento informado de los pacientes y/o sujetos referidos en el artículo. Este documento obra en poder del autor de correspondencia.

\section{Bibliografía}

1. Schecter WP, Hirshberg A, Chang DS, Harris HW, Napolitano LM, Wexner SD, et al. Enteric fistulas: principles of management. J Am Coll Surg. 2009;209:484-91.
2. Majercik S, Kinikini M, White T. Enteroatmospheric fistula: from soup to nuts. Nutr Clin Pract. 2012;27(4):507-12.

3. Arebi N, Forbes A. High-output fistula. Clin Colon Rectal Surg. 2004; 17:89-98.

4. Yetişir $F$, Sarer AE. Operative management of enteroatmospheric fistula in Björck 4 open abdomen patients by the help of laparoscopic lateral approach. Indian J Surg. 2017:79:173-6.

5. DiBaise JK. Management of the short bowel syndrome in adults. UpToDate; 2021. Disponible en: https://www.uptodate.com/contents/management-of-the-short-bowel-syndrome-in-adults

6. DiBaise JK. Pathophysiology of short bowel syndrome. UpToDate; 2021 Disponible en: https://www.uptodate.com/contents/pathophysiology-of-short-bowel-syndrome

7. Gribovskaja-Rupp I, Melton G. Enterocutaneous fistula: proven strategies and updates. Clin Colon Rectal Sur. 2016:29:130-7.

8. Metcalf $\mathrm{C}$. Considerations for the management of enterocutaneous fistula. Br J Nurs. 2019;28:S24-S31.

9. Nikfarjam M, Champagne B, Reynolds HL, Poulose BK, Ponsky JL, Marks JM. Acute management of stoma-related colocutaneous fistula by temporary placement of a self-expanding plastic stent. Surg Innov. 2009;16:270-3.

10. Melich G, Pai A, Balachandran B, Marecik SJ, Prasad LM, Park JJ. Endoscopic control of enterocutaneous fistula by dual intussuscepting stent technique. Surg Endosc. 2016;30:4150-1.

11. Rekstad L, Wasmuth H, Ystgaard B, Stornes T, Seternes A. Topical negative-pressure therapy for small bowel leakage in a frozen abdomen: a technical report. J Trauma Acute Care Surg. 2013;75:487-91.

12. Ruiz-López M. Negative pressure therapy in wounds with enteric fistulas. Cir Esp. 2009;86:29-32.

13. Padar M, Starkopf J, Uusvel G, Reintam Blaser A. Gastrointestinal failure affects outcome of intensive care. J Crit Care. 2019;52:103-8.

14. Rhodes A, Evans LE, Alhazzani W, Levy MM, Antonelli M, Ferrer R, et al. Campaign to Survive Sepsis: international recommendations for the treatment of sepsis and septic shock: 2016. Intensive Care Med. 2017;43:304-77.

15. Puig CA, Waked TM, Baron TH Sr, Wong Kee Song LM, Gutierrez J, Sarr MG. The role of endoscopic stents in the management of chronic anastomotic and staple line leaks and chronic strictures after bariatric surgery. Surg Obes Relat Dis. 2014;10:613-7.

16. Xu ZY, Ren HJ, Huang JJ, Li ZA, Ren JA. Application of a 3D-printed "fistula stent" in plugging enteroatmospheric fistula with open abdomen: a case report. World J Gastroenterol. 2019;25:1775-82.

17. Nguyen NT, Nguyen XM, Dholakia C. The use of endoscopic stent in management of leaks after sleeve gastrectomy. Obes Surg. 2010;20:1289-92.

18. Rebibo L, Wacrenier A, Thiebault H, Delcenserie R, Regimbeau JM. Combined endoscopic and surgical covered stent placement: a new tailored treatment for enteroatmospheric fistula in patients with terminal ileostomy. Endoscopy. 2017;49 (S 01):E35-6. 